\title{
Clinical and Non-Clinical Characteristics of Pulmonary Aspergillosis in Non-Neutropenic Patients
}

\author{
Tingting Chen ${ }^{1}$, Wenfang Tang ${ }^{1}$, Yimin Chen ${ }^{2}$, Jun Zhang ${ }^{1}$ and Xinyou Xie ${ }^{1, *}$ \\ ${ }^{1}$ Sir Run Run Shaw Hospital, Zhejiang University School of Medicine, Hangzhou, China \\ ${ }^{2}$ Zhejiang Provincial Hospital of Traditional Chinese Medicine, Hangzhou, China \\ "Corresponding author: Sir Run Run Shaw Hospital, Zhejiang University School of Medicine, Hangzhou, China. Email: scottxie@zju.edu.cn
}

Received 2019 February 08; Revised 2019 May 13; Accepted 2019 May 17.

\section{Dear Editor,}

Aspergillus is a genus of filamentous, ubiquitous, and opportunistic pathogenic fungi. Patients with underlying lung disease are susceptible to Aspergillus due to their immunodeficiency, but the symptoms become atypical as other lung diseases are contracted (1-3). Aspergillus fumigatus, A. flavus, and A. niger are the primary pathogenic species in most literatures $(4,5)$. The principal forms of pulmonary aspergillosis (PA) are allergic bronchopulmonary aspergillosis (ABPA), chronic (and saprophytic) pulmonary aspergillosis (CPA), and invasive pulmonary aspergillosis (IPA). In non-neutropenic patients, various forms are considered a semi-continuous spectrum of aspergillosis (4). The analysis of the clinical features can deepen our understanding of the spectrum of clinical manifestations of Aspergillus infection and provide references for its diagnosis.

Forty-two PA patients (30 males and 12 females) in the Respiratory Department of our hospital were enrolled in the present study from January 2016 to December 2017. Patients with a hematological disease or granulocytic deficiency were excluded. The average age \pm standard deviation (SD) was $62.45 \pm 16.09$ years. All patients were divided into three groups, including CPA, ABPA, and IPA, according to the consensus on the diagnosis and treatment of pulmonary mycosis (6). Proven IPA was dependent on the histological analysis or positive Aspergillus cultures in sterile sites. Probable IPA met the criteria for a host factor, a clinical criterion, and a mycological criterion, according to the criteria of the European Organization for Research and Treatment of Cancer/Mycoses Study Group (EORTC/MSG) (7). Only cases of proven and probable IPA were included in the IPA group. Clinical features, chest CT, total serum IgE, and Aspergillus species-specific IgE were employed in combination to diagnose ABPA. The underlying lung diseases were comprised of COPD (11/42), bronchiectasis (11/42), TB (6/42), lung cancer (3/42), and in- terstitial lung disease (3/42).

Beta-Glucan (BG) test was performed using a 1-3- $\beta$ D- Glucan test kit (Zhanjiang A\&C Biological Ltd, China), galactomannan (GM) test was conducted with the Platelia Aspergillus EIA test kit (Bio-rad Laboratories, USA), and Creactive protein (CRP) was detected with the CRP test kit (QuikRead go, Finland). Aspergillus was identified by the mass spectrometry (Bruke MALDI Biotype) after being cultured in Sabouraud dextrose agar. Clinical symptoms, chest CT, BG test, GM test, CRP, and fungal cultures were analyzed. Tests were defined as positive when the GM index $\geq 0.5$ and the $1-3-\beta$-D-Glucan (BG test) $\geq 100 \mathrm{pg} / \mathrm{mL}$. Differences between the groups were analyzed using one-way ANOVA test. A P $<0.05$ was the threshold for statistical significance.

The majority of the PA patients presented with cough, expectoration, and chest tightness. Hemoptysis was primarily present in CPA and presumed to be associated with aspergilloma, invading the intercostal artery. Wheezing was primarily present in ABPA and was likely to be associated with a complex hypersensitivity reaction. The IPA patients had the highest rate of fever. The CT findings in CPA, ABPA, and IPA consisted of bronchiectasis $(66.67 \%, 50.00 \%$, and $43.48 \%)$, nodular or mass shadow (66.67\%, 60.00\%, and $34.78 \%$ ), flake shadow $(44.44 \%, 70.00 \%$, and $43.48 \%$ ), cavity $(33.33 \%, 10.00 \%$, and $21.74 \%$ ), pleural thickening (88.89\%, $20.00 \%$, and $56.52 \%)$, lung obstruction collapse $(44.44 \%, 0.00 \%$, and $0.00 \%)$, pulmonary emphysema $(55.56 \%, 40.00 \%$, and $21.74 \%)$, and pulmonary bullae (44.44\%, $0.00 \%$, and $13.04 \%$ ).

Serum $\mathrm{G}$ and $\mathrm{GM}$ tests were performed in all CPA cases, 8 ABPA (8/10), and 22 IPA (22/23). bronchoalveolar lavage fluid (BALF) BG and GM tests were carried out in the remaining patients. No statistically significant differences were established in the serum $\mathrm{BG}$ and GM test values in $\mathrm{CPA}, \mathrm{ABPA}$, and IPA patients $\left(\chi^{2}=2.676, \mathrm{P}=0.262\right.$ and $\chi^{2}=$ $3.166, \mathrm{P}=0.205)$, but the $\mathrm{CRP}$ values were statistically differ- 
ent $\left(\chi^{2}=7.913, P=0.019\right)$ (Table 1$)$. The BALF BG and GM tests were performed in 11 PA patients. Eight patients with negative serum GM underwent BALF GM simultaneously, six of them were positive.

Fungal cultures were positive in none of the CPA cases (0/9), 6 ABPA (6/10), and 14 IPA (14/23). One case of A. flavus and five cases of $A$. fumigatus were identified in the ABPA patients, whereas A. fumigatus was isolated in 14 cases of IPA patients. The number of males was higher than that of females, and the age range was from 50 to 70 years. The clinical characteristics were non-specific. Although the CT findings facilitated an improved PA diagnosis, the imaging results were diverse and non-specific.

The sensitivity of BG and GM tests for the diagnosis of PA in non-neutropenic patients was insufficient. Consistent with Zhou et al. (8), GM antigen levels were greater in BALF as this represented the site of infection. Neutrophils eliminated GM antigens in the serum, which reduced the available levels of detection. Similar to previous studies (9), the median of CRP in IPA patients was the highest, followed in a descending order by that in CPA and ABPA. These findings suggest that CRP could be used in combination with other tests to identify PA.

Sputum fungal cultures were unsuitable for the diagnosis of CPA due to the presence of hyphae that restricted the release into sputum, but the positive rates of the sputum fungal cultures were higher than those of the serum BG and GM in IPA and ABPA. In conclusion, our findings revealed that the symptoms were non-specific. Additionally, the imaging results were diverse, and the microbiological indices were insufficiently sensitive. Therefore, the development and implementation of new indicators or methods to facilitate PA diagnosis are required.

\section{Footnotes}

Authors' Contribution: Jun Zhang and Xinyou Xie conceived and designed the experiments. Tingting Chen and Wenfang Tang performed the experiments. Tingting Chen and Yimin Chen analyzed the data. Tingting Chen contributed to the provision and exploitation of reagents/materials/analysis tools and wrote the manuscript.

Conflict of Interests: The authors declare that they have no conflict of interests.
Ethical Approval: The Ethics Committee of Zhejiang Provincial Hospital of Traditional Chinese Medicine approved this study (Ethical code: 2019-K-300-01).

Financial Disclosure: The authors have no relevant financial interests.

Funding/Support: No funding support.

\section{References}

1. Zarrinfar H, Makimura K, Satoh K, Khodadadi H, Mirhendi H. Incidence of pulmonary aspergillosis and correlation of conventional diagnostic methods with nested PCR and real-time PCR assay using BAL fluid in intensive care unit patients. J Clin Lab Anal. 2013;27(3):181-5 doi: 10.1002/jcla.21580. [PubMed: 23686776].

2. Zarrinfar H, Mirhendi H, Fata A, Khodadadi H, Kordbacheh P. Detection of aspergillus flavus and a. Fumigatus in bronchoalveolar lavage specimens of hematopoietic stem cell transplants and hematological malignancies patients by real-time polymerase chain reaction, nested PCR and mycological assays. Jundishapur J Microbiol. 2015;8(1). e13744. doi: 10.5812/jjm.13744. [PubMed: 25763133]. [PubMed Central: PMC4344768].

3. Zarrinfar H, Mirhendi H, Makimura K, Satoh K, Khodadadi H, Paknejad O. Use of mycological, nested PCR, and real-time PCR methods on BAL fluids for detection of Aspergillus fumigatus and A. flavus in solid organ transplant recipients. Mycopathologia. 2013;176(5-6):377-85. doi: 10.1007/s11046-013-9657-9. [PubMed: 24045934].

4. Kosmidis C, Denning DW. The clinical spectrum of pulmonary aspergillosis. Thorax. 2015;70(3):270-7. doi: 10.1136/thoraxjnl-2014 206291. [PubMed: 25354514].

5. Zarrinfar H, Saber S, Kordbacheh P, Makimura K, Fata A, Geramishoar $\mathrm{M}$, et al. Mycological microscopic and culture examination of 400 bronchoalveolar lavage (BAL) samples. Iran J Public Health. 2012;41(7):70-6. [PubMed: 23113213]. [PubMed Central: PMC3469012].

6. The Society of Respiratory Medicine CSOM. consensus on diagnosis and treatment of pulmonary mycosis. Chin J Tuberc Respir Dis. 2007;11(30):821-34.

7. De Pauw B, Walsh TJ, Donnelly JP, Stevens DA, Edwards JE, Calandra $\mathrm{T}$, et al. Revised definitions of invasive fungal disease from the European Organization for research and treatment of cancer/invasive fungal infections cooperative group and the national institute of allergy and infectious diseases mycoses study group (EORTC/MSG) consensus group. Clin Infect Dis. 2008;46(12):1813-21. doi: 10.1086/588660. [PubMed: 18462102]. [PubMed Central: PMC2671227].

8. Zhou W, Li H, Zhang Y, Huang M, He Q, Li P, et al. Diagnostic value of galactomannan antigen test in serum and bronchoalveolar lavage fluid samples from patients with nonneutropenic invasive pulmonary aspergillosis. J Clin Microbiol. 2017;55(7):2153-61. doi: 10.1128/JCM.00345-17. [PubMed: 28446576]. [PubMed Central: PMC5483917].

9. Roques M, Chretien ML, Favennec C, Lafon I, Ferrant E, Legouge $\mathrm{C}$, et al. Evolution of procalcitonin, C-reactive protein and fibrinogen levels in neutropenic leukaemia patients with invasive pulmonary aspergillosis or mucormycosis. Mycoses. 2016;59(6):383-90. doi: 10.1111/myc.12487. [PubMed: 26931315]. 


\begin{tabular}{|c|c|c|c|c|c|c|}
\hline & $\operatorname{CPA}(n=9)$ & $\operatorname{ABPA}(\mathbf{n}=8)$ & $\operatorname{IPA}(n=22)$ & Total & $\chi^{2}$ & P Value \\
\hline Serum GM test ODI & & & & & 3.166 & 0.205 \\
\hline Median & 0.49 & 0.24 & 0.29 & 0.30 & & \\
\hline Minimum value & 0.19 & 0.18 & 0.13 & 0.13 & & \\
\hline Maximum value & 2.50 & 3.30 & 4.18 & 4.18 & & \\
\hline Serum BG test $(\mathbf{p g} / \mathbf{m L})$ & & & & & 2.676 & 0.262 \\
\hline Median & 37.20 & 24.60 & 78.20 & 53.55 & & \\
\hline Minimum value & 24.80 & 18.20 & 17.40 & 17.40 & & \\
\hline Maximum value & 528.03 & 538.90 & 5000.0 & 5000.00 & & \\
\hline $\mathrm{CRP}(\mathrm{mg} / \mathrm{L})$ & & & & & 7.913 & $0.019^{\mathrm{a}}$ \\
\hline Median & 10.93 & 1.00 & 20.70 & 8.40 & & \\
\hline Minimum value & 1.00 & 0.38 & 0.50 & 0.38 & & \\
\hline Maximum value & 59.67 & 44.62 & 319.58 & 319.58 & & \\
\hline
\end{tabular}

Abbreviations: ABPA: allergic bronchopulmonary aspergillosis; BG test: 1-3- $\beta$-D-Glucan; CPA: chronic pulmonary aspergillosis; CRP: C-reactive protein; GM: galactomannan; IPA: invasive pulmonary aspergillosis; ODI: optical density index.

${ }^{a} \mathrm{P}<0.05$, statistically significant. 